\title{
INFLUENCE OF PSYCHOTIC EPISODES ON GREY MATTER VOLUME CHANGES IN PATIENTS WITH SCHIZOPHRENIA
}

\author{
Antonija Ružić Baršić ${ }^{1,2}$, Gordana Rubeša ${ }^{3,4}$, Diana Mance ${ }^{5}$, Damir Miletić ${ }^{4,6}$, \\ Lea Gudelj ${ }^{3,4}$ \& Ronald Antulov ${ }^{7}$ \\ ${ }^{I}$ Department of Radiology, Thalassotherapia Opatija, Opatija, Croatia \\ ${ }^{2}$ Faculty of Medicine, University Josip Juraj Strossmayer, Osijek, Croatia \\ ${ }^{3}$ Clinic of Psychiatry, Clinical Hospital Centre Rijeka, Rijeka, Croatia \\ ${ }^{4}$ University of Rijeka, Faculty of Medicine, Rijeka, Croatia \\ ${ }^{5}$ University of Rijeka, Department of Physics, Rijeka, Croatia \\ ${ }^{6}$ Department of Clinical Radiology, Clinical Hospital Centre Rijeka, Rijeka, Croatia \\ ${ }^{7}$ Department of Radiology and Nuclear Medicine, Hospital South West Jutland, \\ University Hospital of Southern Denmark, Esbjerg, Denmark
}

received: 17.4.2020;

revised: 21.8.2020;

accepted: 31.8 .2020

\section{SUMMARY}

Background: Schizophrenia is a severe illness whose clinical course is characterized by various numbers of psychotic episodes (PE). The neurotoxic hypothesis (NH) of schizophrenia assumes that psychosis is biologically toxic. The aim of the study was to investigate whether schizophrenia patients $(S P)$ with multiple PE have greater grey matter volume (GMV) reduction compared to $S P$ with fewer PE.

Subjects and methods: We enrolled 106 adult SP and 63 healthy controls. Demographic and clinical data were collected and statistically analysed for all included subjects. Magnetic resonance imaging (MRI) of the brain was acquired on a $1.5 T$ Scanner. SP were grouped according to the number of PE into a group with up to 3 PE (SCHG-1) and with 4 or more PE (SCHG-2). SCHG-1 was further subdivided into two groups regarding to disease duration (DD). Voxel-based morphometry (VBM) analyses were performed between SP groups as well as between SP groups and the healthy controls group (HCG).

Results: No relevant GMV differences were detected between SP groups. Comparison between HCG and SCHG-1 showed only 3 regions with reduced GMV, while multiple regions with reduced GMV were detected when comparing HCG and SCHG-2.

Conclusions: GMV reduction in schizophrenia varies depending on the number of PE when compared to HCG, regardless of disease duration (DD), but PE is not the only contributing factor that leads to neurotoxicity.

Key words: schizophrenia - voxel-based morphometry - grey matter volume - psychotic episode - neurotoxic hypothesis

$$
* * * * *
$$

\section{INTRODUCTION}

The neurodegenerative theory suggests that the main cause of schizophrenia is a neurodegenerative process (Kraepelin et al. 1919, Lieberman 1999), primarily resulting in cortical frontal and prefrontal grey matter (GM) atrophy as well as reduction of pyramidal cells number in the thalamocortical and corticocortical tracts followed by white matter (WM) decrease, probably occurring due to axonal atrophy, genetic disorder of myelin or unrecognized infections (Silva et al. 2019). The before mentioned changes are accompanied by ventricular system enlargement, particularly the lateral ventricles, and increased cerebrospinal fluid (CSF) volume (Keshavan et al. 1998, Lawrie et al. 2002, Horga et al. 2011, Sayo et al. 2012).

The NH is based on the Kraepelinian idea of connection between clinical deterioration and brain damage (Kraepelin et al. 1919, DeLisi 2008). Further on, Wyatt investigated effects of neuroleptic treatment on the natural course of schizophrenia, and suggested that psychosis is biologically toxic resulting with brain tissue degradation with every PE (Wyatt 1991). Neurotoxicity results from repeated exposure to neurochemical stressors
(Lieberman et al. 1997), which are released during PE, and by stress-related hormones that induce functional and structural brain abnormalities leading to neurodegeneration (Wood et al. 2009). The NH was further complemented with findings that the duration of untreated psychosis (DUP) could be neurotoxic and a predictor of poorer disease outcome (Lieberman et al. 1993). DUP may induce neuronal excitotoxic damage, therefore it can be considered a possible explanation for neurotoxicity (Goldberg et al. 2009, McKenzie 2014) DUP can also be understood as one of the neurotoxic insults and has been associated with GMV reduction (Olney \& Farber 1995, Seok Jeong et al. 2005, Gur et al. 1998b).

The clinical course of established schizophrenia is characterized by alternations of PE, remissions and relapses (Kim \& Dilip 2008). Mild and moderate GMV reduction was described in SP with first PE (Zipursky et al. 1998, Shenton et al. 2001). During lifetime some SP have one or few $\mathrm{PE}$, while others have numerous, therefore it could be hypothesized that if every PE is considered as a neurotoxic insult, more prominent GMV loss can be expected in SP with multiple PE while less GMV loss can be expected in SP with few PE. 
Longitudinal MRI studies reported progressive cortical GMV reduction over time in SP (Vita et al. 2012). Schizophrenia shows progression of GMV abnormalities related to DD, where GMV reduction began in the thalami, progressed to the frontal lobes, and then to the temporal and occipital cortices as well and the cerebellum (Jiang et al. 2018). Greater GMV reduction was associated with longer DD and with usage of higher doses of antipsychotic medication (Shenton et al. 2001).

The aim of this study was to test the hypothesis that SP with multiple PE have greater GMV reduction compared to SP with fewer PE, regardless of DD. To the best of this author's knowledge, there are no known publications showing the relationship between the number of PE and GMV changes in SP.

\section{SUBJECTS AND METHODS}

\section{Subjects}

We included 106 adults diagnosed with schizophrenia according to the 10th revision of the International Statistical Classification of Diseases and Related Health Problems (Organization 2004) and Diagnostic and Statistical Manual of Mental Disorders-IV (1994). The SP were recruited from the Psychiatric Clinic, Clinical Hospital Centre Rijeka, Croatia. Clinical assessment was performed by a specially trained psychiatrist (G. R.) who also measured illness severity using the Positive and Negative Syndrome Scale (PANSS) (Kay et al. 1987).
The whole group of $106 \mathrm{SP}$ with schizophrenia was divided into two similar groups according to the number of PE: a group of $61 \mathrm{SP}$ with up to $3 \mathrm{PE}$ (SCHG-1) and a group of 45 SP with 4 or more PE (SCHG-2). Further on, SCHG-1 was subdivided regarding DD: a group of $30 \mathrm{SP}$ with DD up to 3 years (SCHG-1A) and a group of 31 SP with DD of 4 years and longer (SCHG-1B) shown in Figure 1. SCHG-2 has not been divided in subgroups, since the number of SP in the group was too small, thus impairing statistical analysis. The HCG consisted of 63 adult participants, without previous history of psychiatric or neurological illness or head trauma. After a comprehensive description of the study to the subjects, written informed consent was obtained from all participants. The demographic data for all participants is shown in Table 1.

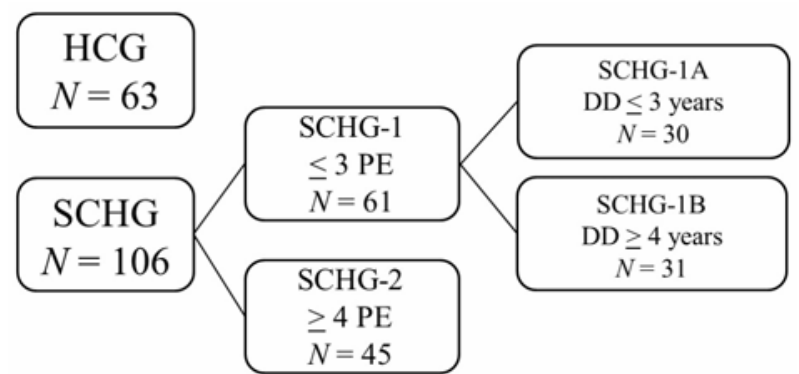

Figure 1. Subject classification: $\mathrm{HCG}$ and SP groups (SCHG-1, SCHG-2, SCHG-1A and SCHG-1B) according to the number of PE and DD

Table 1. Demographic and clinical characteristics of healthy control group (HCG) and schizophrenia patients groups (SCHG)

\begin{tabular}{lccccccccc}
\hline & HCG & SCHG-1 & SCHG-2 & \multicolumn{2}{c}{ Statistical analysis } & SCHG-1A SCHG-1B & \multicolumn{2}{c}{ Statistical analysis } \\
& $(N=63)$ & $(N=61)$ & $(N=45)$ & $t, Z$ or $\chi^{2}$ & $p$ & $(N=30)$ & $(N=31)$ & $F$ or $\chi^{2}$ & $p$ \\
\hline Age (years) & $33(29,40)$ & $30.3 \pm 11.6$ & $43.2 \pm 10.6$ & 32.04 & $<0.0001^{*}$ & $22.6 \pm 6.9$ & $37.7 \pm 10.4$ & 60.82 & $<0.0001^{* *}$ \\
Gender (male/female) & $30 / 33$ & $36 / 25$ & $28 / 17$ & 2.71 & 0.257 & $18 / 12$ & $18 / 13$ & 2.74 & 0.434 \\
Dominant hand (right/ & $59 / 2 / 2$ & $54 / 6 / 1$ & $44 / 1 / 0$ & 3.45 & 0.178 & $27 / 3 / 0$ & $27 / 3 / 1$ & 3.64 & 0.302 \\
left/ambidextrous) & & & & & & & & & \\
Age of DO (years) & & $23.1 \pm 6.8$ & $26.2 \pm 6.9$ & 2.24 & 0.027 & $22.1 \pm 7.1$ & $24.2 \pm 6.4$ & 3.22 & $0.044^{* * *}$ \\
DD (years) & $5(0,12)$ & $17.0 \pm 8.5$ & 5.31 & $<0.0001$ & $0(0,1)$ & $12(9,16)$ & 65.71 & $<0.0001^{+}$ \\
Number of PE & & $2(1,2)$ & $7(5,12)$ & 8.77 & $<0.0001$ & $1(1,2)$ & $2(2,3)$ & 84.08 & $<0.0001^{++}$ \\
PANSS general & & $50.1 \pm 8.2$ & $51.8 \pm 7.9$ & 1.14 & 0.260 & $50.2 \pm 8.9$ & $49.9 \pm 7.5$ & 0.64 & 0.526 \\
\hline
\end{tabular}

Values represent frequency of data; mean \pm standard deviation for normally distributed data; and median with interquartile range for data that are not normally distributed.

HCG - healthy control group; SCHG-1, SCHG-2, SCHG-1A, SCHG-1B - schizophrenia patients groups defined as shown in Figure 1; DO - disease onset; DD - disease duration; PE - psychotic episodes; PANSS - Positive and Negative Syndrome Scale; ${ }^{a}$ HCG, SCHG-1 and SCHG-2; continuous variables were analysed by $t$ - test, Mann-Whitney test or Kruskal-Wallis test (with multiple comparisons of mean ranks as a post hoc analysis) and categorical data by $\chi^{2}$ test;

*post hoc analysis proved that there is statistically significant difference in age between SCHG-2 and other two groups $(p=0.001)$; ${ }^{b}$ HCG, SCHG-1A, SCHG-1B and SCHG-2; continuous variables were analysed by ANOVA (with Tukey HSD test as a post hoc test) or Kruskal-Wallis test (with multiple comparisons of mean ranks as a post hoc analysis) and categorical data by $\chi^{2}$ test; **post hoc analysis proved that there are statistically significant differences in age between SCHG-1A and all the other groups $(p<0.0001)$, as well as between HCG and SCHG-2 $(p<0.003)$;

***post hoc analysis proved that there is significant difference in the age of DO between SCHG-1A and SCHG-2 ( $p=0.035)$; ${ }^{+}$post hoc analysis proved that there are statistically significant differences in DD between SCHG-1A and all the other groups $(p<0.0001)$;

${ }^{++}$post hoc analysis proved that there are statistically significant differences in number of PE between SCHG-2 and all the other groups $(p=0.0001)$. 
All participants gave their signed written informed consent and patients anonymity were preserved. The Ethical Committee of Clinical Hospital Centre Rijeka approved the study, which was performed in accordance with the ethical principles laid down in the seventh and current edition (2013) of the Declaration of Helsinki.

\section{MRI data imaging and processing}

Brain imaging was acquired on a single $1.5 \mathrm{~T}$ Magnetom Avanto Siemens (Erlangen, Germany) using a 32channel head coil and a tilted T1-weighted coronal 3D magnetization prepared rapid acquisition gradient echo sequence (MP-RAGE: TR $2400 \mathrm{~ms}$; TE $3.61 \mathrm{~ms}$; flip angle $8^{\circ}$; FoV $240 \times 240 \mathrm{~mm}$; matrix $192 \times 192$; two acquisitions). This sequence produced 160 contiguous images (slice thickness $1.2 \mathrm{~mm}$ ), sagittal orientation.

The whole-brain images pre-processing and data voxel-based morphometry (VBM) analysis was done with the VBM8 toolbox (http://dbm.neuro.uni-jena.de/ $\mathrm{vbm} /$ ) using the SPM12 software package (http://www.fil.ion.ucl.ac.uk/spm/) running on MATLAB 9.0 (The MathWorks, Natick, MA, U.S.A.). After segmentation into GM, white matter (WM) and CSF, the GM and WM segments of all subjects were normalized to create an average anatomical template. DARTEL registration of the GM and WM segments for all the subjects was performed on the template to create individual deformation fields (IDF). The IDF of each subject was used to nonlinearly spatially register the GM segments on the template. Finally, the modulated normalized nonlinear GM segments were smoothed by an 8-mm full-width at half-maximum Gaussian kernel.

\section{Statistical analysis}

Group differences in demographic and clinical characteristics were assessed with Statistica 12 (StatSoft Inc., USA). Categorical variables were tested through $\chi^{2}$ test, while continuous data was first tested for normality by Kolmogorov-Smirnov test. The descriptive statistics for data that are normally distributed are presented with arithmetic mean and standard deviation, while data that do not follow normal distribution are presented with the median and interquartile range shown in Table 1 . The significance of differences between two groups of normally distributed data was tested by $t$-test for independent data, while for testing the difference of three and more groups of normally distributed data analyses of variance (ANOVA) and Tukey honestly significant difference test for post hoc analysis were used. In case of two groups of data that are not normally distributed, the Mann-Whitney test was applied, and for comparison between three and more groups KruskalWallis test was used as well as multiple comparisons of mean ranks for post hoc analysis. The $p<0.05$ value was selected for the level of statistical significance.

To detect regional GMV differences between the groups we used VBM toolbox (http://dbm.neuro.unijena.de/vbm) in SPM8 (https://www.fil.ion.ucl.ac.uk/ spm/software/spm8/). Age and sex were retained as nuisance variables, and an image threshold of 0.2 was used. During the analysis of clinical data, we observed group differences in the age of disease onset (DO) and $\mathrm{DD}$, so those variables were also used as covariates in all comparisons that included SCHG-1 and SCHG-2. In the same manner, the age of DO and number of PE were used as covariates in all comparisons concerning SCHG$1 \mathrm{~A}$ and SCHG-1B. Cluster level was set at $\mathrm{k} \geq 20$ voxels for the whole brain analysis. All analyses were performed at $p<0.05$ significance level with family wise error as a correction for multiple comparisons. Coordinates of significant clusters were converted from Montreal Neurological Institute space to Talairach space using GingerALE (https://www.brainmap.org/ale/). Talairach labels for significant clusters were generated by Talairach Client using a nearest GM search (http://www.talairach.org/client.html). Results were visualized using $\mathrm{xjView}$ toolbox (http://www.alivelearn.net/xjview).

\section{RESULTS}

\section{Demographic and clinical characteristics}

SCHG-2 were older than subjects in both HCG and SCHG-1. SCHG-1 had DO at younger age, shorter DD and fewer PE than SCHG-2 subjects. Further analyses proved that SCHG-1A is the youngest of all examined groups and has shorter DD than SP in SCHG-1B and SCHG-2. Also, SCHG-1A had earlier age of DO than SCHG-2. Both SCHG-1A and SCHG-1B had less PE than SCHG-2. There was no statistically significant difference in the PANSS (general) score between the investigated groups.

\section{Voxel-based morphometry: between group analysis}

There were no detectable statistically significant GMV differences when subgroups SCHG-1, SCHG-2, SCHG-1A and SCHG-1B were compared with each other.

A comparison between HCG and SCHG-1 revealed decreased GMV in the right precentral gyrus, right insula and left claustrum in SCHG-1 shown in Table 2 and Figure 2. Right transversal temporal gyrus was the only detected region with statistically significant GMV difference in the HCG and SCHG-1A comparison. Analysis of HCG relative to SCHG-1B resulted in statistically significant GMV differences in right precentral gyrus and right claustrum shown in Table 2 . An increased number of brain regions with statistically significant GMV differences were detected by comparison of HCG and SCHG-2: left medial frontal gyrus, left middle frontal gyrus, left medial frontal gyrus, right superior frontal gyrus, left inferior frontal gyrus, right insula, left middle temporal gyrus, right superior temporal gyrus, right middle temporal gyrus, left superior temporal gyrus, left anterior cingulate gyrus, left claustrum, right culmen and left and right declive shown in Table 2 and Figure 3. 
Table 2. Results of VBM analyses of GMV differences between HCG and SP divided according to Figure 1

\begin{tabular}{|c|c|c|c|c|c|c|}
\hline \multirow[b]{2}{*}{ Anatomical region } & \multirow{2}{*}{$\begin{array}{l}\text { nber of voxels } \\
\text { in cluster } \\
k \\
\end{array}$} & \multicolumn{2}{|c|}{ Statistics } & \multicolumn{3}{|c|}{$\begin{array}{l}\text { Coordinates of voxel with maximal } \\
\text { statistical significance (Talairach) }\end{array}$} \\
\hline & & $t$ & $p$ & $x$ & $y$ & $z$ \\
\hline \multicolumn{7}{|l|}{ HCG vs. SCHG-1 } \\
\hline Frontal Right, Precentral gyrus, BD 6 & 305 & 5.96 & $<0.0001$ & 47.51 & -9.27 & 6.91 \\
\hline Sub-lobar Right, Insula, $B D 13$ & 502 & 5.72 & 0.001 & 30.88 & 14.31 & 11.56 \\
\hline Sub-lobar, Left, Claustrum & 28 & 4.83 & 0.027 & -31.64 & 8.79 & 12.68 \\
\hline \multicolumn{7}{|l|}{ HCG vs. SCHG-1A } \\
\hline Temporal Right, Transversal temporal gyrus, BD 41 & 117 & 5.80 & $<0.0001$ & 48.78 & -21.11 & 12.56 \\
\hline \multicolumn{7}{|l|}{ HCG vs. SCHG-1B } \\
\hline Frontal Right, Precentral gyrus, $B D 6$ & 43 & 5.26 & 0.005 & 46.12 & -7.86 & 7.02 \\
\hline Sub-lobar Right, Claustrum & 63 & 4.81 & 0.029 & 28.09 & 15.60 & 12.99 \\
\hline \multicolumn{7}{|l|}{ HCG vs. SCHG-2 } \\
\hline Limbic Left, Anterior cingulate, BD 32 & 508 & 5.78 & 0.001 & 0.62 & 34.34 & -4.62 \\
\hline Posterior Lobe Left Cerebellum, Declive & 255 & 5.48 & 0.002 & -23.21 & -61.83 & -15.49 \\
\hline Posterior Lobe Right Cerebellum, Declive & 110 & 5.48 & 0.002 & 22.6 & -66.13 & -16.47 \\
\hline Frontal Left, Middle frontal gyrus, $B D 8$ & 34 & 5.45 & 0.002 & -25.13 & 20.59 & 50.4 \\
\hline Limbic Left, Anterior cingulate, $B D 32$ & 107 & 5.29 & 0.004 & 0.23 & 34.26 & 25.9 \\
\hline Temporal Right, Superior temporal gyrus, BD 22 & 136 & 5.26 & 0.005 & 55.80 & -9.57 & 9.72 \\
\hline Frontal Left, Medial frontal gyrus, $B D 10$ & 49 & 5.24 & 0.005 & 0.61 & 53.51 & 1.25 \\
\hline Sub-lobar Right, Insula, $B D 13$ & 114 & 5.19 & 0.006 & 32.39 & 22.08 & 4.21 \\
\hline Temporal Right, Middle temporal gyrus, BD 22 & 37 & 5.18 & 0.007 & 54.58 & -12.45 & -4.09 \\
\hline Anterior Lobe Right Cerebellum, Culmen & 43 & 5.16 & 0.007 & 21.22 & -49.69 & -12.23 \\
\hline Temporal Left, Superior temporal gyrus, BD 22 & 27 & 5.15 & 0.008 & -52.46 & -10.28 & 6.47 \\
\hline Sub-lobar Left, Claustrum & 158 & 5.14 & 0.008 & -31.64 & 7.39 & 12.55 \\
\hline Frontal Left, Medial frontal gyrus, $B D 6$ & 28 & 5.04 & 0.012 & -18.31 & -11.84 & 50.14 \\
\hline Temporal Left, Middle temporal gyrus, BD 21 & 20 & 5.01 & 0.013 & -55.14 & -21.92 & -4.14 \\
\hline Frontal Right, Superior frontal gyrus, BD 10 & 29 & 4.84 & 0.026 & 26.75 & 48.88 & 18.82 \\
\hline Frontal Left, Inferior frontal gyrus & 20 & 4.79 & 0.031 & -35.52 & 42.39 & 0.94 \\
\hline
\end{tabular}

HCG - healthy controls group; SP - schizophrenia patients; SCHG-1-SP group with up to 3 episodes; SCHG-1A - SP with up to 3 episodes and DD up to 3 years; SCHG-1B - SP with up to 3 PE and DD longer than 4 years; SCHG-2 - SP with 4 or more PE

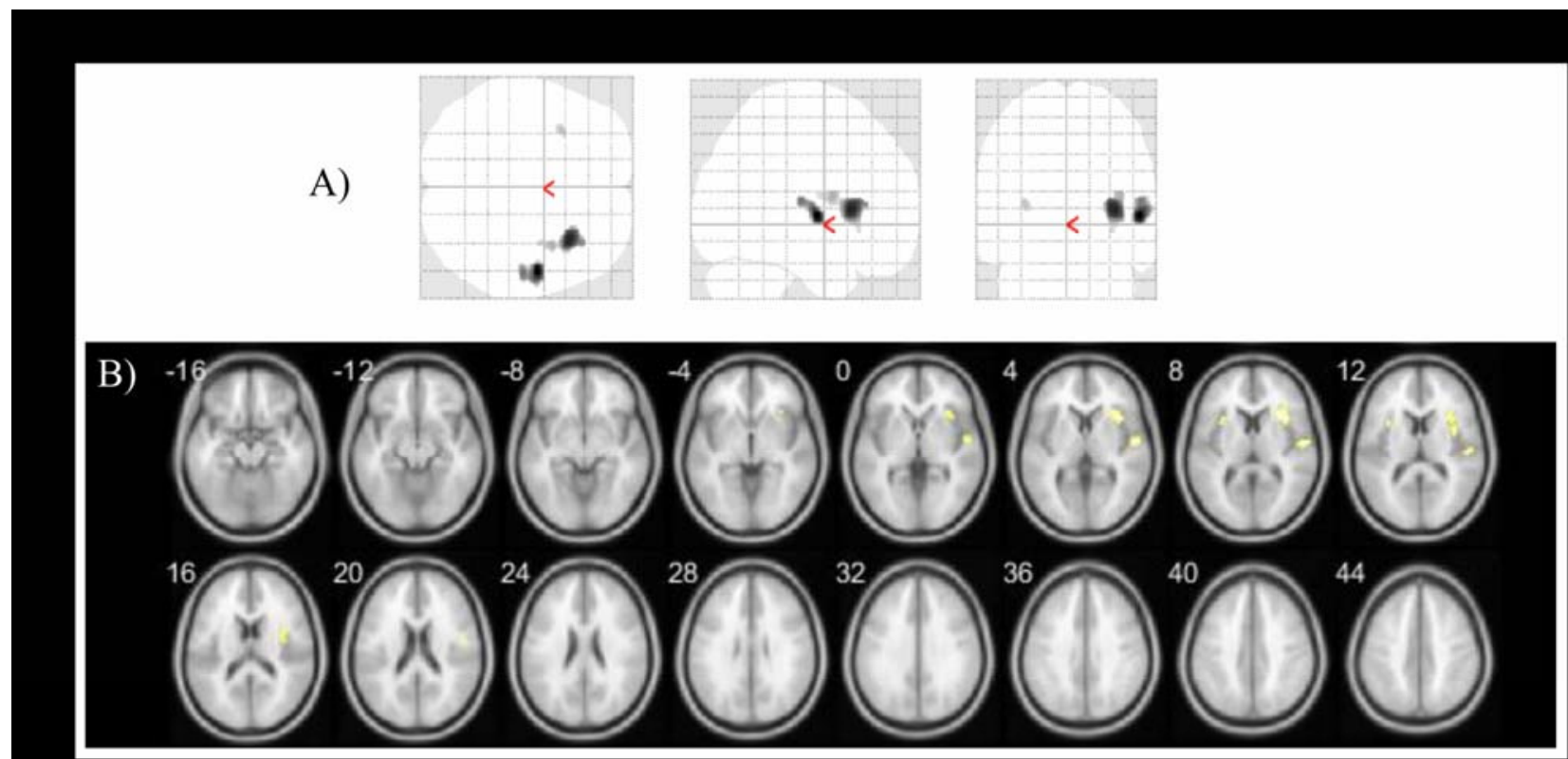

Figure 2. Results of GMV of analysis between HCG and SCHG-1. The yellow-coloured areas indicate brain regions with significantly reduced GMV relative to HCG. The statistical threshold was set at $\mathrm{p}<0.05 \mathrm{FWE}$ corrected; minimum cluster size $=20$. (A) glass view, (B) slice view 


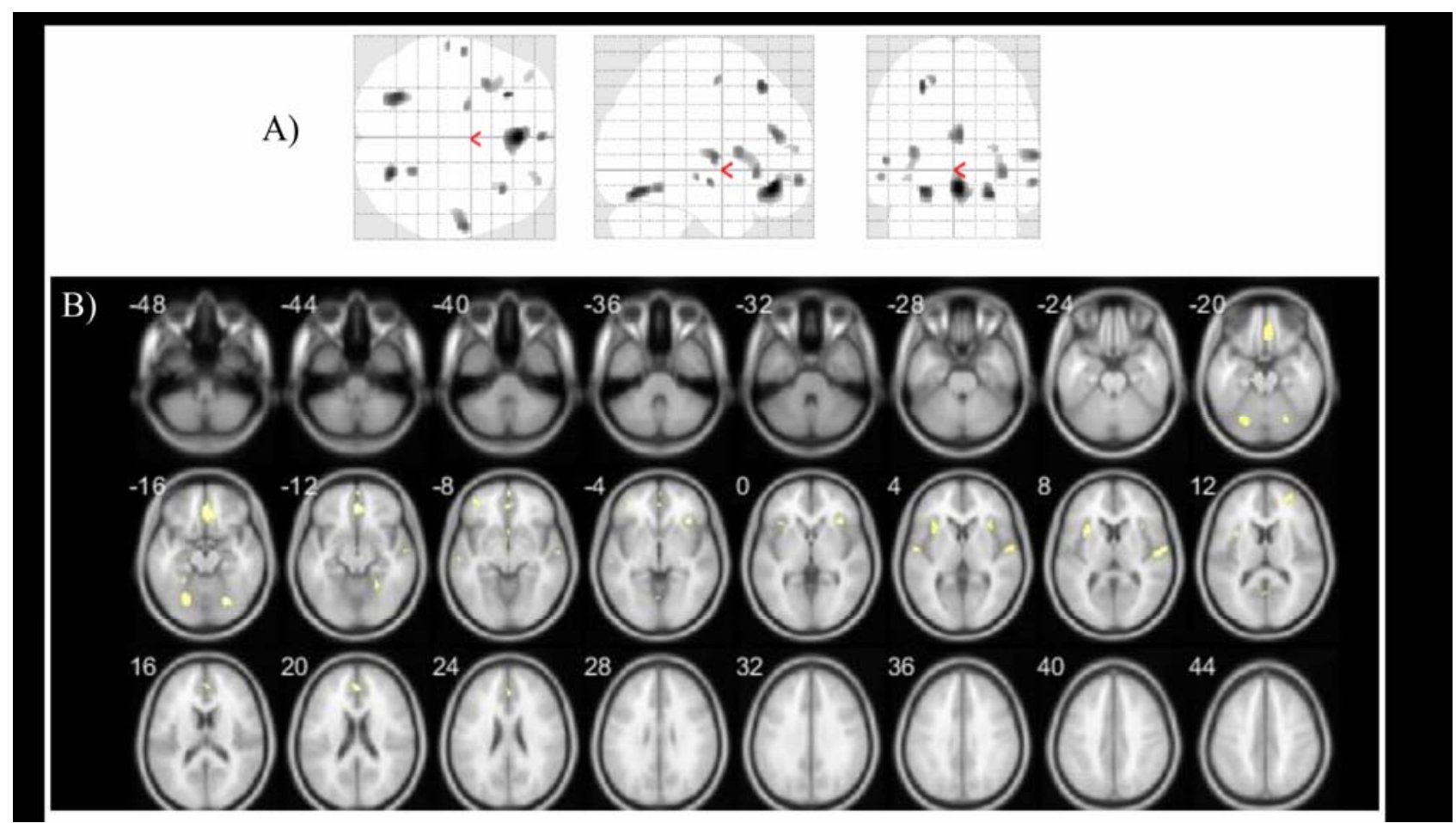

Figure 3. Results of GMV of analysis between HCG and SCHG-2. The yellow-coloured areas indicate brain regions with significantly reduced GMV relative to HCG. The statistical threshold was set at $\mathrm{p}<0.05$ FWE corrected; minimum cluster size $=20$. (A) glass view, (B) slice view

\section{DISCUSSION}

Our study analyzed the possible influence of the number of PE on GMV in SP. When SP were divided into subgroups according to the number of PE and compared to HCG the findings demonstrated that the number, extension and distribution of affected regions differ. SCHG-1 had only few reduced GMV regions, while SCHG-2 had many regions with reduced GMV. The only common anatomical regions showing GMV reduction in SCHG-1 and SCHG-2 are insula and claustrum. Insular changes contribute to many sensory deficits found in schizophrenia (Wylie \& Tregellas 2010) and in chronic SP right insular cortex changes were observed (Nesvag et al. 2008). GMV reduction in insula and claustrum might contribute to positive symptoms in schizophrenia (Roiz-Santianez et al. 2010). Considering the fact that insular thinning is present in early phases of schizophrenia and that it is independent of intervening variables, there is a possibility that changes in insula might be considered as a biological marker for schizophrenia (Shah et al. 2017).

Primary regions of GMV abnormalities in schizophrenia are gyrus rectus, superior temporal gyrus, left hippocampal cortex and insular cortex (Shah et al. 2017). Our study also revealed abnormalities in these regions in SCHG-2, but not SCHG-1. In addition to the above-mentioned regions the most affected brain region in SCHG-2 is the anterior cingulate cortex. Abnormalities in this region are related with cognitive deficits in schizophrenia (Szeszko et al. 2000, McGuire et al. 1998), and thinning of the anterior segment of cingulate cortex is reported to be connected with illness severity and DD (Wang et al. 2007).

Decreased GMV in the cerebellum is associated with a decline in dynamic functional connectivity between the cerebellum and the frontoparietal network in SP (He $\&$ Luo 2019). Reduced GMV in vermis and cerebellar tonsils were found in SP with cognitive deficits in first PE (Wang et al. 2017). In our study only SCHG-2 had cerebellar GMV reduction. On the contrary, an influence of number of PE on cerebellar GMV reduction was not observed in recent studies (Yeganeh-Doost et al. 2011, Moberget et al. 2018).

More prominent GMV changes were found when comparing $\mathrm{HCG}$ with SCHG-2 in relation to the comparison between HCG and SCHG-1, regardless of DD, which could be suggestive of progressive GMV loss related to the number of PE. This finding may represent the confirmation of $\mathrm{NH}$ of schizophrenia; therefore PE could be considered a neurochemical stressor (Lieberman et al. 1997).

Lately, a staging model for schizophrenia has been proposed (Agius et al. 2010, Wood et al. 2011). McGorry`s recognized eight stages of illness and observed GMV reduction in frontal cortex, superior temporal lobe and corpus callosum in the early phase of schizophrenia that progressed as DD increased (McGorry et al. 2010). Many authors found that longer DD and progressive brain changes in chronic schizophrenia are associated with GMV reductions in superior temporal gyrus and in frontal cortex (Chan et al. 2009, DeLisi et al. 2004, Gur et al. 1998a, Mathalon et al. 2001, van Haren et al. 2008, van Haren et al. 2007). 
Both SCHG-1 subgroups, SCHG-1A and SCHG-1B, regardless of DD had only one or two regions respectively with GMV reduction when compared to HCG which implicates that the number of PE plays an important role, possibly even more important than DD. Our study did not reveal any GMV difference when schizophrenia groups and subgroups were compared with each other. This might be due to heterogeneity of the factors responsible for neurotoxicity among the groups as well as specific factors for each SP.

Length of DUP is associated with neurotoxicity (Marshall et al. 2005, Perkins et al. 2005), where longer DUP leads to worse cognitive deterioration (Rund 2014). Potential mechanism of neurotoxicity in DUP is dopaminergic hyperactivity which can induce neuronal apoptosis during PE (Crespo-Facorro et al. 2007, Simantov et al. 1996, Anderson et al. 2014). Sometimes, it is hard to determine the exact length of DUP because the compliance of SP is variable (Anderson et al. 2014, Malla et al. 2002). DUP was not observed in our study.

Previously widely used conventional antipsychotics such as haloperidol have been often producing extrapyramidal side effects resulting in neurotoxicity (Ukai et al. 2004), necrosis and apoptosis (Behl et al. 1995, Noh et al. 2000). On the contrary, clinical studies showed advantages of atypical antipsychotics, resulting in neuroprotection, neurotrophins production and neurogenesis (Wakade et al. 2002, He et al. 2009). Medication adherence is also variable in psychiatric patients, including SP. It can vary from complete nonadherence to partial adherence and complete adherence (Svestka \& Bitter 2007, Higashi et al. 2013). Nonadherence to medication has an impact on DD, length of PE, on relapses, rehospitalization and consequently on neurotoxicity (Leucht \& Heres 2006). Our SP were treated with both conventional and atypical antipsychotics, and medication usage was not observed in this study. Therefore, a major limitation to our study is that we did not observe all the contributing factors which can lead to neurotoxicity.

On the other hand, the nonexistence of GMV differences when schizophrenia groups and subgroups were compared with each other could be explained by how the groups were formed. Our grouping aimed for a similar number of SP in each category, therefore it could be postulated that future studies could detect GMV differences between SP if other criteria for grouping according to the number of $\mathrm{PE}$ would be applied, while keeping the groups large enough for comparison.

\section{CONCLUSIONS}

We found that the number of PE results in neurotoxicity and has more impact on GMV changes than $\mathrm{DD}$, but the number of PE is not the only factor leading to neurotoxicity. Therefore, further studies should include SP groups with more homogenous distribution of factors responsible for neurotoxicity.

\section{Acknowledgements:}

The authors kindly thank all participants for their contribution. We thank the radiographers at Department of Clinical Radiology, Clinical Hospital Centre Rijeka, Croatia for their technical expertise and support in data acquisition.

\section{Conflict of interest: None to declare.}

\section{Contribution of individual authors:}

Antonija Ružić Baršić: writing original draft, formal analysis, investigation, conceptualization.

Gordana Rubeša: writing - review and editing, resources, supervision, conceptualization.

Diana Mance: validation, writing original draft, software, methodology.

Damir Miletić: supervision, writing - review and editing. Lea Gudelj: resources, writing - review and editing.

Ronald Antulov: writing - review and editing, software, methodology, supervision.

\section{References}

1. Diagnostic and statistical manual of mental disorders: DSM-IV. American Psychiatric Association, Washington DC, 1994

2. Agius M, Goh C, Ulhaq $S$ \& McGorry P: The staging model in schizophrenia, and its clinical implications. Psychiatr Danub 2010; 22:211-220

3. Anderson KK, Voineskos A, Mulsant BH, George TP \& McKenzie KJ: The role of untreated psychosis in neurodegeneration: a review of hypothesized mechanisms of neurotoxicity in first-episode psychosis. Can J Psychiatry 2014; 59:513-517

4. Behl C, Rupprecht R, Skutella T\& Holsboer F: Haloperidol-induced cell death - mechanism and protection with vitamin $E$ in vitro. Neuroreport 1995; 7:360-364

5. Chan WY, Chia MY, Yang GL, Woon PS, Sitoh YY, Collinson et al.: Duration of illness, regional brain morphology and neurocognitive correlates in schizophrenia. Ann Acad Med Singapore 2009; 38:388-388

6. Crespo-Facorro B, Roiz-Santianez R, Pelayo-Teran JM, Gonzalez-Blanch C, Perez-Iglesias R, Gutiérrez A et al.: Caudate nucleus volume and its clinical and cognitive correlations in first episode schizophrenia. Schizophr Res 2007; 91:87-96

7. DeLisi LE: The concept of progressive brain change in schizophrenia: implications for understanding schizophrenia. Schizophr Bull 2008; 34:312-321

8. DeLisi LE., Sakuma M, Maurizio AM, Relja $M \&$ Hoff AL: Cerebral ventricular change over the first 10 years after the onset of schizophrenia. Psychiatry Res 2004; 130:57-70

9. Goldberg TE, Burdick KE, McCormack J, Napolitano B, Patel RC, Sevy SM et al.: Lack of an inverse relationship between duration of untreated psychosis and cognitive function in first episode schizophrenia. Schizophr Res 2009; 107:262-266

10. Gur RE, Cowell P, Turetsky BI, Gallacher F, Cannon T, Bilker $W$ et al.: A follow-up magnetic resonance imaging study of schizophrenia. Relationship of neuroanatomical 
changes to clinical and neurobehavioral measures. Arch Gen Psychiatry 1998a; 55:145-152

11. Gur RE, Maany V, Mozley PD, Swanson C, Bilker $W$ \& Gur RC: Subcortical MRI volumes in neuroleptic-naive and treated patients with schizophrenia. Am J Psychiatry $1998 b ; 155: 1711-1717$

12. He $H$ \& Luo C: Reduction in gray matter of cerebellum in schizophrenia and its influence on static and dynamic connectivity. 2019; 40:517-528

13. He J, Kong J, Tan QR \& Li XM: Neuroprotective effect of atypical antipsychotics in cognitive and non-cognitive behavioral impairment in animal models. Cell adhesion \& migration 2009; 3:129-137

14. Higashi K, Medic G, Littlewood KJ, Diez T, Granstrom O \& De Hert M: Medication adherence in schizophrenia: factors influencing adherence and consequences of nonadherence, a systematic literature review. Ther $A d v$ Psychopharmacol 2013; 3:200-218

15. Horga G, Bernacer J, Dusi N, Entis J, Chu K, Hazlett EA et al.: Correlations between ventricular enlargement and gray and white matter volumes of cortex, thalamus, striatum, and internal capsule in schizophrenia. European archives of psychiatry and clinical neuroscience 2011; 261:467-476

16. Jiang Y, Luo C, Li X, Duan M, He H, Chen $X$ et al.: Progressive Reduction in Gray Matter in Patients with Schizophrenia Assessed with MR Imaging by Using Causal Network Analysis. Radiology 2018; 287:729

17. Kay SR, Fiszbein A \& Opler LA: The Positive and Negative Syndrome Scale (PANSS) for Schizophrenia. Schizophrenia Bulletin 1987; 13:261-276

18. Keshavan MS, Haas GL, Kahn CE, Aguilar E, Dick EL, Schooler NR, et al.: Superior temporal gyrus and the course of early schizophrenia: progressive, static, or reversible? J Psychiatr Res 1998; 32:161-167

19. Kim $T$ \& Dilip V: Clinical Handbook of Schizophrenia. Guilgord Publications, 2008

20. Kraepelin E, Robertson GM \& Barclay RM: Dementia praecox and paraphrenia. Chicago Medical Book Co. Chicago, 1919

21. Lawrie SM, Whalley HC, Abukmeil SS, Kestelman JN, Miller P, Best JJ et al.: Temporal lobe volume changes in people at high risk of schizophrenia with psychotic symptoms. Br J Psychiatry 2002; 181:138-143

22. Leucht $S$ \& Heres $S$ : Epidemiology, clinical consequences, and psychosocial treatment of nonadherence in schizophrenia. J Clin Psychiatry 2006; 67 Suppl 5:3-8

23. Lieberman J, Jody D, Geisler S, Alvir J, Loebel A, Szymanski $S$ et al.: Time course and biologic correlates of treatment response in first-episode schizophrenia. Arch Gen Psychiatry 1993; 50:369-376

24. Lieberman JA: Is schizophrenia a neurodegenerative disorder? A clinical and neurobiological perspective. Biol Psychiatry 1999; 46:729-739

25. Lieberman JA, Sheitman BB \& Kinon BJ: Neurochemical sensitization in the pathophysiology of schizophrenia: deficits and dysfunction in neuronal regulation and plasticity. Neuropsychopharmacology 1997; 17:205-229

26. Malla AK, Norman RM, Manchanda R, Ahmed MR, Scholten D, Harricharan $R$ et al.: One year outcome in first episode psychosis: influence of DUP and other predictors. Schizophr Res 2002; 54:231-242

27. Marshall M, Lewis S, Lockwood A, Drake R, Jones $P$ \& Croudace T: Association between duration of untreated psychosis and outcome in cohorts of first-episode patients: a systematic review. Arch Gen Psychiatry 2005; 62:975-983

28. Mathalon DH, Sullivan EV, Lim KO \& Pfefferbaum A: Progressive brain volume changes and the clinical course of schizophrenia in men: a longitudinal magnetic resonance imaging study. Arch Gen Psychiatry 2001; 58:148-157

29. McGorry PD, Nelson B, Goldstone S \& Yung AR: Clinical staging: a heuristic and practical strategy for new research and better health and social outcomes for psychotic and related mood disorders. Can J Psychiatry 2010; 55:486-497

30. McGuire PK, Quested DJ, Spence SA, Murray RM, Frith $C D \&$ Liddle PF: Pathophysiology of 'positive' thought disorder in schizophrenia. Br J Psychiatry 1998; 173:231235

31. McKenzie KJ: How does untreated psychosis lead to neurological damage? Canadian journal of psychiatry. Revue canadienne de psychiatrie 2014; 59:511-512

32. Moberget T, Doan NT, Alnaes D, Kaufmann T, CordovaPalomera A, Lagerberg TV et al.: Cerebellar volume and cerebellocerebral structural covariance in schizophrenia: a multisite mega-analysis of 983 patients and 1349 healthy controls. Mol Psychiatry 2018; 23:1512-1520

33. Nesvag R, Lawyer G, Varnas K, Fjell AM, Walhovd KB, Frigessi A et al.: Regional thinning of the cerebral cortex in schizophrenia: effects of diagnosis, age and antipsychotic medication. Schizophr Res 2008; 98:16-28

34. Noh JS, Kang HJ, Kim EY, Sohn S, Chung YK, Kim SU et al.: Haloperidol-induced neuronal apoptosis: role of p38 and c-Jun-NH(2)-terminal protein kinase. $J$ Neurochem 2000; 75:2327-2334

35. Olney JW \& Farber NB: Glutamate receptor dysfunction and schizophrenia. Arch Gen Psychiatry 1995; 52:9981007

36. Perkins DO, Gu H, Boteva $K$ \& Lieberman JA: Relationship between duration of untreated psychosis and outcome in first-episode schizophrenia: a critical review and meta-analysis. Am J Psychiatry 2005; 162:1785-1804

37. Roiz-Santianez $R$, Perez-Iglesias $R$, Quintero $C$, Tordesillas-Gutierrez D, Mata I, Ayesa R et al.: Insular cortex thinning in first episode schizophrenia patients. Psychiatry Res 2010; 182: 216-222

38. Rund BR: Does active psychosis cause neurobiological pathology? A critical review of the neurotoxicity hypothesis. Psychol Med 2014; 44:1577-1590

39. Sayo A, Jennings RG \& Van Horn JD: Study factors influencing ventricular enlargement in schizophrenia: a 20 year follow-up meta-analysis. Neurolmage 2012; 59:154-167

40. Seok Jeong B, Kwon JS, Yoon Kim S, Lee C, Youn T, Moon $\mathrm{CH}$ et al.: Functional imaging evidence of the relationship between recurrent psychotic episodes and neurodegenerative course in schizophrenia. Psychiatry Res 2005; 139:219-228

41. Shah C, Zhang W, Xiao Y, Yao L, Zhao Y, Gao X et al.: Common pattern of gray-matter abnormalities in drugnaive and medicated first-episode schizophrenia: a multimodal meta-analysis. Psychol Med 2017; 47:401413

42. Shenton ME, Dickey CC, Frumin $M \&$ McCarley $R W: A$ review of MRI findings in schizophrenia. Schizophr Res 2001; 49:1-52 
43. Silva AI, Haddon JE, Ahmed Syed Y, Trent S, Lin TE, Patel $Y$ et al.: Cyfip1 haploinsufficient rats show white matter changes, myelin thinning, abnormal oligodendrocytes and behavioural inflexibility. Nat Commun 2019; 10:3455

44. Simantov R, Blinder E, Ratovitski T, Tauber M, Gabbay M \& Porat S: Dopamine-induced apoptosis in human neuronal cells: inhibition by nucleic acids antisense to the dopamine transporter. Neuroscience 1996; 74:39-50

45. Svestka $J \&$ Bitter I: Nonadherence to antipsychotic treatment in patients with schizophrenic disorders. Neuro Endocrinol Lett 2007; 28 Suppl 1:95-116

46. Szeszko PR, Bilder RM, Lencz T, Ashtari M, Goldman RS, Reiter $G$ et al.: Reduced anterior cingulate gyrus volume correlates with executive dysfunction in men with firstepisode schizophrenia. Schizophr Res 2000; 43:97-108

47. Ukai W, Ozawa H, Tateno M, Hashimoto E \& Saito T: Neurotoxic potential of haloperidol in comparison with risperidone: implication of Akt-mediated signal changes by haloperidol. J Neural Transm (Vienna) 2004; 111:667-681

48. van Haren NE, Hulshoff Pol HE, Schnack HG, Cahn W, Brans $R \&$ Carati I: Progressive brain volume loss in schizophrenia over the course of the illness: evidence of maturational abnormalities in early adulthood. Biol Psychiatry 2008; 63:106-113

49. van Haren NE, Hulshoff Pol HE, Schnack HG, Cahn W, Mandl RC, Collins DL et al.: Focal gray matter changes in schizophrenia across the course of the illness: a 5year follow-up study. Neuropsychopharmacology 2007; 32:2057-2066

50. Vita A, De Peri L, Deste G \& Sacchetti E: Progressive loss of cortical gray matter in schizophrenia: a metaanalysis and meta-regression of longitudinal MRI studies. Transl Psychiatry 2012; 2:e190
51. Wakade CG, Mahadik SP, Waller JL \& Chiu FC: Atypical neuroleptics stimulate neurogenesis in adult rat brain. $J$ Neurosci Res 2002; 69:72-79

52. Wang J, Zhou L, Cui C, Liu Z \& Lu J: Gray matter morphological anomalies in the cerebellar vermis in firstepisode schizophrenia patients with cognitive deficits. BMC Psychiatry 2017; 17:374

53. Wang L, Hosakere M, Trein JC, Miller A, Ratnanather JT, Barch DM et al.: Abnormalities of cingulate gyrus neuroanatomy in schizophrenia. Schizophr Res 2007; 93:66-78

54. Wood SJ, Pantelis C, Yung AR, Velakoulis D \& McGorry $P D$ : Brain changes during the onset of schizophrenia: implications for neurodevelopmental theories. Med J Aust 2009; 190:S10-13

55. Wood SJ, Yung AR, McGorry PD \& Pantelis C: Neuroimaging and treatment evidence for clinical staging in psychotic disorders: from the at-risk mental state to chronic schizophrenia. Biol Psychiatry 2011; 70:619-625.

56. World Health Organization: ICD-10: international statistical classification of diseases and related health problems. World Health Organization, 10th revision, 2nd ed. 2004

57. Wyatt RJ: Neuroleptics and the natural course of schizophrenia. Schizophr Bull 1991; 17:325-351

58. Wylie KP \& Tregellas JR: The role of the insula in schizophrenia. Schizophr Res 2010; 123:93-104

59. Yeganeh-Doost P, Gruber O, Falkai P \& Schmitt A: The role of the cerebellum in schizophrenia: from cognition to molecular pathways. Clinics (Sao Paulo, Brazil) 2011; 66 Suppl 1:71-77

60. Zipursky RB, Lambe EK, Kapur $S$ \& Mikulis DJ: Cerebral gray matter volume deficits in first episode psychosis. Arch Gen Psychiatry 1998; 55:540-546

Correspondence:

Ronald Antulov, MD

Department of Radiology and Nuclear Medicine, Hospital South West Jutland,

University Hospital of Southern Denmark

Finsensgade 35, 6700 Esbjerg, Denmark

E-mail: ronald.antulov@outlook.com 